La Revue

des Droits

de l'Homme

\section{La Revue des droits de l'homme}

Revue du Centre de recherches et d'études sur les droits fondamentaux

Actualités Droits-Libertés | 2014

\title{
Le « milieu du gué » de la protection législative des lanceurs d'alerte
}

Lanceur d'alerte (Liberté d'expression)

Anna Billard, Marc Duranton, Jean-Philippe Foegle et Tristan MartinTeodorczyk

\section{(2) OpenEdition}

\section{Journals}

Édition électronique

URL : http://journals.openedition.org/revdh/752

DOI : $10.4000 /$ revdh.752

ISSN : 2264-119X

Éditeur

Centre de recherches et d'études sur les droits fondamentaux

Référence électronique

Anna Billard, Marc Duranton, Jean-Philippe Foegle et Tristan Martin-Teodorczyk, «Le « milieu du gué » de la protection législative des lanceurs d'alerte », La Revue des droits de l'homme [En ligne], Actualités Droits-Libertés, mis en ligne le 20 mai 2014, consulté le 30 avril 2019. URL : http://

journals.openedition.org/revdh/752 ; DOI : 10.4000/revdh.752

Ce document a été généré automatiquement le 30 avril 2019.

Tous droits réservés 


\title{
Le " milieu du gué » de la protection législative des lanceurs d'alerte
}

Lanceur d'alerte (Liberté d'expression)

\author{
Anna Billard, Marc Duranton, Jean-Philippe Foegle et Tristan Martin- \\ Teodorczyk
}

1 «M. Hammarberg souligne, en citant les mémoires d'Andreï Sakharov, que la menace d'une révélation freine les atteintes aux droits de l'homme. Lorsque les signalements responsables sont protégés, par la loi et par des mesures pratiques, on peut s'attendre à ce que les violations des droits de l'homme diminuent" (Paul Stephenson, Michael Levi, "La protection des donneurs d'alerte ", Rapport d'étude sur la faisabilité d'un instrument juridique sur la protection des employés qui divulguent des informations dans l'intérêt public, Conseil de l'Europe, CDCJ(2012)9FIN, Strasbourg, décembre 2012, p. 40). Initialement envisagée dans le secteur privé comme un simple «mode de gestion » de l'entreprise parmi d'autres, la protection des lanceurs d'alerte « tend à revêtir une juridicité de plus en plus affirmée aisément perceptible à l'occasion de certains contentieux» (Paul-Henri Antonmattei, Paul Vivien "Chartes éthiques, alerte professionnelle et droit du travail français : état des lieux et perspectives ", Rapport remis au Ministre du travail Gérard Larcher, La Documentation française, mars 2007, p. 39.)

2 Entendu comme « toute personne soucieuse de tirer la sonnette d'alarme afin de faire cesser des agissements pouvant représenter un risque pour autrui » (Resolution 1729 (2010), Protection of "whistle-blowers") le porteur (ou lanceur) d'alerte tend en effet à s'inscrire durablement dans le paysage juridique et politique français. Si les lanceurs d'alerte les plus médiatiques (tels Edward Snowden) sont ceux qui sont dirigés vers une instance extérieure (vers la presse notamment), la plupart des lanceurs d'alerte sont des lanceurs d'alerte "internes", c'est-à-dire qu'ils révèlent des dysfonctionnements au sein de leur propre organisation (Bowden P., 2005, "A comparative analysis of whistleblower protection ", Australian Association for Professional and Applied Ethics, 12th Annual Conference, 28-30 September). Cette distinction entre lanceurs d'alerte tend toutefois à perdre de sa pertinence au regard de l'évolution de la jurisprudence 
européenne relative à la liberté d'expression. Celle-ci prévoit en effet que la diffusion des informations s'opère "d'abord auprès de son supérieur ou d'une autre autorité ou instance compétente», avant que ne s'envisage la divulgation au public "en cas d'impossibilité manifeste d'agir autrement» (Cour EDH, G.C. 12 février 2008, GUJA c. Moldavie, Req. $\mathrm{n}$ - 14277/04, § 142) ; dès lors, si une distinction existe bel et bien entre lanceurs d'alerte « internes » et « externes ", elle ne constitue nullement une summa divisio, et ne recoupe donc, au mieux, qu'une notion de gradation dans l'utilisation des canaux d'alerte.

Les réflexions menées par le Conseil de l'Europe sur ce sujet depuis quelques années (voir la Résolution 1729 \$1 (2010) Protection des «donneurs d'alerte »), en particulier celles faisant suites aux révélations d'Edward Snowden sur le programme de surveillance généralisé des métadonnées, réalisé par la NSA (Assemblée Parlementaire, Proposition de résolution sur la surveillance et les lanceurs d' alerte, 6 août 2013, Doc. 13288), tendent à envisager de manière de plus en plus explicite le whistleblowing comme l'un des moyens privilégiés de favoriser l'effectivité des droits de l'homme ${ }^{1}$. La résolution précitée de l'Assemblée Parlementaire du Conseil de l'Europe précise d'ailleurs à cet égard que «la définition des révélations protégées doit inclure tous les avertissements de bonne foi concernant divers actes illicites, $y$ compris toutes les violations graves des droits de l'homme, qui affectent ou menacent la vie, la santé, la liberté et tout autre intérêt légitime des individus ».

4 Au-delà, les divers textes internationaux protégeant les porteurs d'alerte tendent à s'harmoniser et à promouvoir une approche "globale » du phénomène des lanceurs d'alerte. La Convention civile sur la corruption du Conseil de l'Europe du 4 novembre 1999 prévoit ainsi en son article 9 la protection des employés signalant des faits de corruption, tandis que la Convention des Nations Unies contre la corruption du 31 octobre 2003 prévoit l'obligation pour tout agent de signaler toute activité, tout emploi, tout avoir ou don d'où pourrait résulter un conflit d'intérêt (\$5). S'agissant des canaux de signalement, l'article 13.2 de la même convention prévoit la mise en place d'organes de prévention connus du public et accessibles pour tout signalement d'infraction « $y$ compris sous-couvert d'anonymat ». L'article 33 prévoit enfin la protection de «toute personne qui signale aux autorités compétentes, de bonne foi et sur la base de soupçons raisonnables » des faits de corruption. Cette approche globale (global approach) du phénomène, doublée par la mise en place d'une législation complète (comprehensive legislation) s'entend, aux yeux de nombreux membres de la doctrine, comme l'un des éléments-clés d'une législation efficiente en matière de promotion du whistleblowing (David Lewis, "Arbitrary legal protection will only deter whistleblowers.", Nursing Standard 27.39 (2013) : 31-31). Pour le juriste australien A.J Brown, «le caractère complet d'une législation protectrice des lanceurs d'alerte, quel que soit le secteur couvert, dépend de trois critères: l'étendue des faits susceptibles d'être signalés; l'étendue et la diversité des secteurs d'activité couverts par la législation; et, enfin, l'étendue des personnes pouvant bénéficier du processus et des protections prévues par la loi.» (Brown, A. J., "Towards 'ideal'whistleblowing legislation? Some lessons from recent Australian experience.", E-Journal of International and Comparative Labour Studies (2013).

5 Plusieurs pays, en particulier l'Australie, les Etats-Unis et le Royaume-Uni, qui font figure d'États pionniers sur ce point, adoptent une conception globale du lanceur d'alerte ${ }^{2}$. Ainsi, pour ne prendre qu'une exemple, en Australie, le Public Interest Disclosure Act de 2013 adopte une définition très large des faits pouvant justifier le déclenchement d'une alerte, près de 21 types de comportements répréhensibles étant définis (PID 
Act 2013 (Cth), s 29.). Il en va de même pour le nombre de secteurs d'activité couverts par la législation, définie de manière très large et non limitative, et qui couvre la quasitotalité des activités de l'Etat, à l'exclusion notable et regrettable toutefois de celle des juges et membres du Parlement (PID Act 2013 (Cth), ss 29, 30 et 32). Enfin, au sein des personnes pouvant bénéficier des processus et des protections prévues par la loi, figurent, de manière extensive, à la fois les employés, les contractants de l'administration, les stagiaires, mais également les consommateurs et plus largement les citoyens (PID Act 2013 (Cth), ss 29, 30, and 31(b)).

6 L'approche française du phénomène du lancement d'alerte est quand à elle loin d'atteindre la précision et la complétude de certaines législations ou même de correspondre aux standards dictés par le droit international et européen. Malgré les progrès notables réalisés en 2013 en matière de protection des lanceurs d'alerte, le droit français actuel reste marqué par une approche parcellaire et partielle du phénomène, sans qu'une réelle mise en cohérence ne soit envisagée. Peut-on mesurer l'étendue des progrès réalisés? Outre la très timide consolidation du statut du fonctionnaire lanceur d'alerte que nous n'aborderons pas (voir, sur ce sujet: ADL du 13 mars 2014 par Serge Slama et Jean-Philippe Foegle), une progression à pas de loup se laisse voir dans trois domaines principaux.

7 Première loi en date pour l'année 2013, la loi du 16 avril sur l'alerte sanitaire et environnementale a confirmé l'attention particulière accordée aux lanceurs d'alerte sanitaires et environnementaux. Ceux-ci bénéficient désormais d'un véritable statut protecteur, bien que des zones d'ombres persistent $\left(\mathbf{1}^{\circ}\right)$. La protection du salarié et du fonctionnaire lanceur d'alerte a, elle aussi, connu quelques progrès : la loi du 6 décembre 2013 relative à la fraude fiscale et la grande criminalité économique et financière, ainsi que la loi du 11 octobre 2013 relative à la transparence de la vie publique en sont deux exemples. Cette évolution reste néanmoins mesurée, puisque surtout limitée à la dénonciation de faits de corruption ou de conflits d'intérêts $\left(2^{\circ}\right)$. Enfin, la question de la protection des sources journalistiques, vitale pour la protection des porteurs d'alerte « externes », connaîtra sans doute en 2014 une évolution, qui s'avère toutefois d'ores et déjà bien décevante $\left(3^{\circ}\right)$.

\section{$1 \%$ - La progression encourageante du statut des lanceurs d'alerte sanitaires et environnementaux}

8 La thématique de l'alerte sanitaire, liée aux crises sanitaires intervenues lors de la dernière décennie (amiante, nucléaire, risque radioactif, vache folle) qui a constitué la «porte d'entrée » de la réflexion sur la protection des «lanceurs d'alerte » en France. (Christine Noiville et Marie-Angèle Hermitte, « Quelques pistes pour un statut juridique du chercheur lanceur d'alerte »). Bien que l'essor des droits environnementaux, doublée de préoccupations de "démocratie environnementale » aient conduit à une émergence progressive de la figure du "lanceur d'alerte sanitaire et environnemental», (A) la construction d'un statut protecteur ad hoc pour ces personnes s'est faite de manière récente et relativement laborieuse. (B) 


\section{A - La construction juridique progressive d'un acteur de la démocratie environnementale et sanitaire}

9 La nécessité d'une protection des lanceurs d'alerte sanitaires et environnementaux apparaît d'autant plus essentielle que ceux-ci sont susceptibles de dévoiler des faits correspondant à la violation de droits fondamentaux nouvellement consacrés, notamment le "droit à santé » et le "droit à un environnement sain ", désormais érigés au rang constitutionnel ${ }^{3}$. L'alerte environnementale est, plus sûrement encore, liée à l'émergence $d u$ "principe de précaution». Introduite en droit français par la loi «Barnier » du 2 février 1995 et codifié à l'article 110-1 du code de l'environnement, cette norme a en outre été constitutionnalisée par la révision du 1er mars 2005 ayant annexé la Charte de l'environnement à la Constitution (voir l'article 5 de la Charte)..

Or, une simple lecture sommaire du principe de précaution permet de saisir la proximité conceptuelle de cette notion avec celle d'alerte environnementale. Toutes deux tendent, en effet, vers le même but : rendre public et permettre le traitement d'un risque futur en train d'advenir, sans disposer nécessairement de tous les éléments pour convaincre. La mission Lepage de 2008, qui s'était prononcée en faveur de la mise en place d'une protection pour les lanceurs d'alerte, avait d'ailleurs explicitement lié les deux notions. Pour la mission, le lancement d'une alerte «devrait avoir pour deuxième effet de mettre en ceuvre le principe de précaution et de suspendre la possibilité pour l'entreprise ou le secteur d'activité dans laquelle alerte est lancée d'invoquer le risque de développement ». Surtout, le lancement d'une alerte «devrait avoir pour effet (...) de générer des crédits de recherche pour permettre de savoir le plus rapidement possible si l'alerte est justifiée ou si elle ne l'est pas» (Corinne Lepage, Mission Lepage, Rapport final phase 1. 2008). Ce principe semble donc imposer non seulement une protection de la parole du lanceur d'alerte, mais également la mise en place de procédures de traitement effectif de l'alerte.

11 En outre, la protection de la parole des scientifiques lanceurs d'alerte apparait également comme une déclinaison logique du droit à la liberté d'expression dans le domaine des sciences. Dans une affaire «Hertel» relative à l'interdiction faite au requérant de publier des articles consacrés aux dangers pour la santé des fours à micro-ondes, la Cour a relevé que «la mesure en cause a [...] pour effet de censurer partiellement les travaux de ce dernier [le requérant] et de limiter grandement son aptitude à exposer publiquement une thèse qui a sa place dans un débat public dont l'existence ne peut être niée ». Soulignant qu'«il serait particulièrement excessif de limiter la liberté d'expression à l'exposé des seules idées généralement admises » (Cour EDH, 25 août 1998, Hertel c. Suisse, req. n 53440/99), la Cour de Cassation a, quant à elle, mené un raisonnement similaire dans l'affaire «Cicolella », en mettant en balance les exigences de la «libre-recherche scientifique », et les intérêts de l'employeur. Dans son arrêt du 11 octobre 2000, la Cour de cassation a en effet relevé que l'exercice du pouvoir de direction de l'employeur à l'égard des scientifiques doit s'exercer «dans le respect des responsabilités confiées à l'intéressé et de l'indépendance due aux chercheurs » (Cass. soc. 11 oct. $2000 \mathrm{n}^{\circ} 3716$, pourvoi $\left.\mathrm{n}^{\circ} 98-45.276.\right)^{4}$

Nonobstant le fait que nombre de droits fondamentaux viennent au secours et à l'appui de la parole du scientifique lanceur d'alerte, l'effectivité du droit du scientifique d'alerter sur les risques environnementaux ou sanitaires est faible. En effet, qu'il travaille dans le domaine public ou dans une entreprise privée, celui-ci reste soumis à des devoirs limitant sa liberté de parole (secret industriel, secret commercial, devoir de loyauté), qui 
paraissent ontologiquement contraires à la «logique d'insoumission» présidant au déclenchement d'alerte (Leclerc Olivier. «La protection du salarié lanceur d'alerte », in «Au cœur des combats juridiques. Pensées et témoignages de juristes engagés », 2007, 287-298). Dans le secteur public, ce sont les obligations déontologiques du fonctionnaire qui font obstacle à la protection effective des lanceurs d'alerte (ADL du 13 mars 2014, op. cité). Dans le secteur privé, le scientifique porteur d'alerte soumis au pouvoir de direction de l'employeur s'expose, encore plus gravement, à des mesures de rétorsion de sa part. Nous étudierons cette problématique de manière plus approfondie dans la suite de cet article (voir infra, partie II, A)

\section{B - Une mise en place laborieuse d'un statut protecteur des lanceurs d'alerte sanitaires et environnementaux}

13 La mise en place d'un tel statut s'est pourtant faite de manière laborieuse. En effet, ce n'est qu'au travers de la loi n 2011-2012 du 29 décembre 2011 relative au renforcement de la sécurité sanitaire du médicament et des produits de santé que furent posées les bases d'un statut juridique du «lanceur d'alerte »: est notamment prévue à l'article L. 5312-4-2 du code de la santé publique, une protection contre la discrimination de la personne ayant «relaté ou témoigné, de bonne foi, soit à son employeur, soit aux autorités judiciaires ou administratives de faits relatifs à la sécurité sanitaire des produits mentionnés à l'article L. 5311-1 dont elle aurait eu connaissance dans l'exercice de ses fonctions». La charge de la preuve est en outre inversée, et repose ici sur l'employeur.La loi $n^{\circ}$ 2013-316 du 16 avril 2013 relative à l'indépendance de l'expertise en matière de santé et d'environnement et à la protection des lanceurs d'alerte change relativement la donne en offrant aux lanceurs d'alerte des garanties supplémentaires sur deux plans : une garantie de protection de leur liberté d'expression contre les mesures de rétorsion de leur employeur d'une part; une garantie de traitement effectif de l'alerte d'autre part.

En premier lieu, la loi crée pour la première fois un véritable statut, particulièrement complet, du lanceur d'alerte sanitaire et environnemental. L'article premier de la loi dispose en effet que «toute personne physique ou morale a le droit de rendre publique ou de diffuser de bonne foi une information concernant un fait, une donnée ou une action, dès lors que la méconnaissance de ce fait, de cette donnée ou de cette action lui parait faire peser un risque grave sur la santé publique ou sur l'environnement. L'information qu'elle rend publique ou diffuse doit s'abstenir de toute imputation diffamatoire ou injurieuse ». La diffusion d'une information, qui peut passer par la voie hiérarchique, mais également par voie de presse, devient un véritable «droit ", dont le champ d'application se veut, d'après les propos mêmes du rapporteur du projet de loi à l'Assemblée Nationale Jean Roumégas, particulièrement complet, ayant vocation à couvrir «non seulement les salariés mais également les agents publics » (Jean-Louis Roumégas. Rapport $n^{\circ} 650$ fait au nom de la commission des affaires sociales sur la proposition de loi, adoptée par le Sénat, relative à l'indépendance de l'expertise en matière de santé et d'environnement et à la protection des lanceurs d'alerte, 23 janvier 2013). S'agissant de la notion de bonne foi, le rapporteur pour avis sur le projet de loi précise à cet égard que celle-ci correspond à la « conviction de se trouver dans une situation conforme au droit, avec la conscience d'agir sans léser les droits d'autrui ». 
15 L'usage par les lanceurs d'alerte de leur liberté d'expression est, à l'instar de ce qui existe en matière de protection des lanceurs d'alerte dans le cadre des lois précitées sur le médicament, protégé par un article 12 prévoyant la nullité de tout licenciement motivé par le fait d'«avoir relaté ou témoigné, de bonne foi, soit à son employeur, soit aux autorités judiciaires ou administratives, des faits entrant dans le champ d'application de l'article $1 »$. La charge de la preuve est ici aussi inversée et repose encore une fois sur l'employeur. Enfin, selon toute logique, le contrôle des modalités d'usage de la parole par les lanceurs d'alerte, nécessaire pour éviter des dérives ${ }^{5}$, est naturellement calqué sur le contrôle de droit commun prévu par le droit pénal de la presse. Dans ce cadre, respectueux du droit au procès équitable, la personne soupçonnée d'imputation diffamatoire est «admise à produire, pour les nécessités de sa défense, les pièces de nature à établir la vérité des faits ou sa bonne foi, sans qu'elles puissent être écartées des débats au motif qu'elles auraient été obtenues par des moyens illicites ou déloyaux » ( Cass.Crim, 19 janvier 2010, Bull. crim. 2010, n 12).

En second lieu, la loi apporte de très sérieuses garanties s'agissant du traitement effectif de l'alerte. L'article 2 de la loi institue ainsi une Commission nationale de la déontologie et des alertes en matière de santé publique et d'environnement, commission qui sera chargée de «veiller aux règles déontologiques s'appliquant à l'expertise scientifique et technique et aux procédures d'enregistrement des alertes en matière de santé publique et d'environnement ». Il appartiendra notamment à cette commission de définir les critères de recevabilité des alertes (alinéa 3) et de transmettre aux ministres concernés les alertes dont elle sera saisie (al. 4). Il lui appartiendra également de se prononcer sur les codes de déontologie mis en place dans les établissements publics, et elle pourra par ailleurs être saisie par un nombre très large de personnes, dont, notamment, les associations de défense de l'environnement, les organisations syndicales et certains ordres professionnels. La composition de cette commission est fixée par l'article 5 de la loi: ces membres, qui ne sont pas encore nommés, demeurant logiquement soumis à des règles de "confidentialité, d'impartialité et d'indépendance dans l'exercice de leur fonction». Les décisions prises sur la déontologie de l'expertise devraient, en toute logique au regard d'une décision récente du conseil d'Etat (CE, 27 avr. 2011, Formindep, $\mathrm{n}^{\circ}$ 334396), être prises en compte pour apprécier les obligations déontologiques des experts scientifiques. Surtout, les canaux de l'alerte sont désormais clarifiés, et de nombreuses garanties sont apportées pour permettre aux lanceurs d'alerte de ne pas voir leur signalement " enterré " par leur employeur. L'article 8 de la loi crée en effet un art. L. 4133-1 au sein du code du travail, qui dispose que «le travailleur alerte immédiatement l'employeur s'il estime, de bonne foi, que les produits ou procédés de fabrication utilisés ou mis en œuvre par l'établissement font peser un risque grave sur la santé publique ou l'environnement ». Cette alerte peut également, par la création d'un article L. 4133-2 du code du travail, transiter via le représentant du personnel au comité d'hygiène, de sécurité et des conditions de travail; dans cette hypothèse, l'employeur « examine la situation conjointement avec le représentant du personnel au comité d'hygiène, de sécurité et des conditions de travail qui lui a transmis l'alerte et l'informe de la suite qu'il réserve à celle-ci ».

17 L'alerte prévue au sein de l'entreprise, qui passe par la voie hiérarchique, est dotée de deux garanties de traitement effectif. La première est liée à l'intervention d'un tiers dans la relation binaire employé-employeur puisqu'en l'absence de suite dans un délai d'un mois ou en cas de divergence sur le bien fondé d'une alerte, l'article L 4133-3 du code 
du travail prévoit la possibilité pour le travailleur ou son représentant de saisir le représentant de l'Etat dans le département, qui pourra alors transmettre l'alerte aux autorités compétentes et prendre les mesures nécessaires pour protéger le salarié. La seconde est liée à la sanction de l'employeur saisi d'une alerte qui ne prendrait aucune mesure; celui-ci, précise l'article 13 de la loi, ne pourra bénéficier de l'exonération de responsabilité prévue au $4^{\circ}$ de l'article 1386-11 du code civil.

Si ces évolutions vont dans le bon sens, il ne faudrait toutefois pas exagérer leur portée effective. L'on peut d'une part déplorer que les modalités d'exercice de l'alerte soient à nouveau renvoyées à du "droit mou ", dont l'effectivité reste problématique. Ainsi l'ambitieuse proposition de créer dans chaque entreprise de plus de onze salariés des " cellules d'alerte sanitaires et environnementales » qui se seraient vues octroyées un "droit d'enquête », en association avec le comité d'hygiène, de sécurité et des conditions de travail (CHSCT) et un droit de saisine de la Haute Autorité, a été supprimé. Sans doute aurait-il fallu plus d'ambitions dans la création de cette autorité ad hoc, et surtout permettre aux salariés une saisine directe de celle-ci. La saisine d'une instance extérieure apparait en effet nettement moins dissuasive que la voie hiérarchique. Au-delà, cette loi n'aborde pas un élément pourtant essentiel dans la mise en place d'une culture ouverte à l'alerte : celle de la nécessité d'aménager -et non de supprimer- les règles relatives au secret défense et au secret industriel. Cette problématique était pourtant abordée de manière claire et tranchée par la mission Lepage de $2008^{6}$. Celle-ci proposait notamment, en s'inspirant des législations précitées, de créer une obligation de communication de toutes les études préalables et les données sous-jacentes à la délivrance d'une autorisation dans la mesure où elles concernent la santé ou l'environnement (proposition 8) et d'isoler au sein des documents classés secret défense les informations confidentielles pour permettre de créer une version publique de ceux-ci (proposition 10). En l'absence d'une réelle réflexion sur ces thématiques, les lanceurs d'alerte environnementaux seront toujours amenés à se heurter à la loi du secret.

\section{$2 \%$ Protection des salariés lanceurs d'alerte: une évolution limitée au signalement d'infractions et de conflits d'intérêt}

19 Le lanceur d'alerte professionnel jouit d'une reconnaissance qui oscille entre institutionnalisation croissante et atomisation persistante ; son statut juridique balance entre autonomisation et rattachement à d'autres branches du droit. Toutes proportions gardées, ces remarques tendent ainsi à rapprocher le lanceur d'alerte de ce qui constituerait une nouvelle Cassandre : astreint à faire connaitre une certaine forme de l'avenir, sans pour autant qu'une protection, in fine un statut spécifique, ne lui soit accordé. En effet, ni le droit commun du travail (A), ni les protections spécifiques, trop restrictives (B), ne semblent à même de leur offrir la protection qui leur est nécessaire.

\section{A - Cassandre mérite-t-elle protection ? Le difficile cheminement du droit d'alerte au sein du droit commun du travail}


21 Par delà ces quelques propos liminaires, on remarque qu'historiquement prévaut une relative indifférence quant aux lanceurs d'alerte professionnels. Ou, pour le dire autrement, « on constate une faible propension des salariés du secteur privé à dénoncer les fraudes internes aux entreprises ou les pratiques corruptrices auxquelles ses représentants pourraient se livrer » (Rapport annuel du Service Central de Prévention de la Corruption pour l'année 2011, chapitre V, p. 220). De fait, la situation n'était « historiquement » envisagée qu'au travers de cas limitativement énumérés, notamment par le code du travail ; lequel éludait la question au travers de l'obligation de signalement de harcèlement sexuel et moral, ou via l'obligation de dénonciation des mesures discriminatoires. En outre, cette prise en charge textuelle pour le moins parcellaire se trouva consolidée non seulement par la jurisprudence (voir en ce sens Cass. Soc. 14 mars 2000, 97-43.268), mais encore et surtout par l'ensemble des règles de procédures, qui se montrent tout autant adaptées aux contentieux classiques qu'elles se révèlent peu enclines à embrasser les particularités des dispositifs d'alerte professionnels (constitution de partie civile délicate, risque de dénonciation calomnieuse, conditions restrictives tenant à la protection des témoins, etc.).

Ces obligations éparses de dénoncer semblent entrer en contradiction avec la nature même de la relation de travail, relation déséquilibrée s'il en est. Rappelons que le salarié, soumis à une obligation générale de discrétion (Cass. Soc., 5 mai 1997, CSPB, 1997, S. 91) est tenu de ne pas divulguer les informations dont il a connaissance dans l'exercice de ses fonctions, et ce ni à l'extérieur ni à l'intérieur de l'entreprise (Cass. Soc., 30 juin 1982 : Bull. civ. 1982, V, n 314). Les employeurs peuvent au surplus insérer au sein du contrat de travail des clauses de confidentialité destinées à protéger le savoirfaire de l'entreprise, qui peuvent s'appliquer après la fin du contrat de travail (Cass. soc., 19 mars 2008: RJS 2008, $n^{\circ}$ 631). Cette obligation de confidentialité semble fort heureusement devoir s'effacer d'elle-même dans les hypothèses où l'alerte porte sur un fait délictueux, dans le cas où celui-ci est révélé à des interlocuteurs habilités à la recevoir (Cass. Soc., 14 mars 2000, Bull.Civ. V, n ${ }^{\circ} 104$; RJS, 4/00, n 388). Le problème demeure toutefois entier lorsque la divulgation d'informations est directement assortie d'une sanction pénale, comme dans le cas d'une violation du secret professionnel.

Plus problématique encore est le cas où un lanceur d'alerte révèlerait publiquement des faits utiles au débat public qui ne pourraient être considérés comme directement et manifestement délictueux ${ }^{7}$ Dans toutes les hypothèses, la validité de l'obligation de confidentialité imposée au salarié doit répondre à un "intérêt légitime» de l'entreprise et être "proportionnée " au but recherché ${ }^{8}$. Cette appréciation renvoie donc à une difficile conciliation entre les droits fondamentaux du salarié d'une part, et les droits de l'entreprise d'autre part. Or, on peut craindre que pour des raisons d'opportunité économique, l'équilibre tende vers la primauté des droits de l'entreprise. D'où la nécessité urgente d'un statut ad hoc pour les lanceurs d'alerte.

Un embryon de statut spécifique pour les lanceurs d'alerte est heureusement apparu, dès le début des années 2000, sous l'impulsion du droit international et du droit nordaméricain. Suite entre autres à la chute de l'entreprise Enron, les pouvoirs publics américains adoptèrent le Sarbanes-Oxley Act (SOX), une loi fédérale entrée en vigueur le 30 juillet 2002 et qui eut pour effet d'institutionnaliser la procédure d'alerte éthique dans les entreprises Nord-Américaines, mais également dans leurs filiales. Fut alors assumé le principe d'une protection des lanceurs d'alerte, et notamment «l'obligation pour les entreprises américaines et leurs filiales de mettre en place des codes de conduite internes et un 
système de surveillance collective par déclenchement d'alerte afin de pallier la défaillance des systèmes de contrôle interne des entreprises » (SCPC, op. cité, p. 225). Dès lors, le SOX attira de manière certaine l'attention de grandes entreprises, ainsi que leurs filiales, sur les procédures d'éthique, et "a contribué directement ou indirectement à leur existence, leur évolution et (...) leur publicité » (Didier, Christelle. "L'alerte professionnelle en France : un outil problématique au coeur de la RSE." 4e colloque du RIODD (Réseau international de recherche sur les Organisations et le Développement Durable), 2009).

Pour autant, la CNIL assujettît temporairement la traduction en France de dispositifs d'alerte à de nouvelles considérations liées aux droits de la personne. Au sein d'une première délibération prise en 2005 (délibération n 2005-110 du 26 mai 2005 relative à une demande d'autorisation de McDonald's France pour la mise en œuvre d'un dispositif d'intégrité professionnelle), elle n'hésita pas à assimiler de tels procédés à des " systèmes organisés de délation professionnelle»: était particulièrement visé ici l'anonymat probable des alertes. Par la suite, dans un deuxième document destiné à assouplir sa position, et qui résumerait à lui seul les hésitations dont font l'objet les lanceurs d'alerte salariés, la Commission publia le 10 novembre 2005 (document d'orientation pour la mise en œuvre de dispositifs d'alerte professionnelle conformes à la loi du 6 janvier 1978) un vademecum conforme à la fois aux préconisations de la pratique internationale et de la loi du 6 janvier 1978 relative à l'informatique, aux fichiers et à la liberté. Précisant "qu'elle n'a pas d'opposition de principe à de tels dispositifs dès lors que les droits des personnes mises en cause (...) dans une alerte sont garantis au regard des règles relatives à la protection des données personnelles ", la CNIL annonçait également la mise en place d'un régime d'autorisation unique (délibération n ${ }^{\circ}$ 2005-305 du 8 décembre 2005 portant autorisation unique de traitements automatisés de données à caractère personnel mis en œuvre dans le cadre de dispositifs d'alerte professionnelle) ; ledit système offrant aux entreprises qui le souhaitent un système simplifié d'instauration d'alerte professionnelle.

26 Au delà de cette position de la CNIL, constatons que le cadre législatif a quelque peu évolué depuis. En témoigne la loi nº 2007-1598 relative à la lutte contre la corruption, qui visait à transposer en droit français la Convention des Nations Unies contre la corruption du 31 octobre 2003 (convention dite «De Mérida»). Celle-ci a inséré dans le code du travail un article L1161-1 protégeant de manière spécifique le lanceur d'alerte salarié, en le protégeant contre toute mesure discriminatoire, notamment en inversant la charge de la preuve en la matière et en prévoyant la nullité de tout licenciement. Néanmoins, cet article fait l'objet d'une mise en œuvre restrictive (voir en ce sens, Cass. Soc. 8 décembre 2009, 08-17.191), étant donné que l'article ne s'applique qu'aux signalements d'infraction de corruption. En définitive, les différentes considérations susmentionnées témoignent d'un cadre juridique à la fois verrouillé foisonnant et peu cohérent.. Qui plus est, il convient de relever que, de l'aveu même de la CNIL, le dispositif mis en place " ne correspond pas à la réalité sociale des entreprises françaises » (SCPC, op. cité, p. 231). C'est donc dans ce contexte que sont intervenues les deux lois de la fin 2013.

\section{B - L'évolution limitée de la protection des lanceurs d'alerte en 2013}

27 Ces deux lois (loi n $2013-907$ du 11 octobre 2013 relative à la transparence de la vie publique et Loi du 6 décembre 2013 relative à la lutte contre la fraude fiscale et la grande 
délinquance économique et financière) permettent en effet à la France d'accoucher d'un système, certes encore parcellaire, de protection des salariés et fonctionnaires lanceurs d'alerte. Celui-ci reste toutefois limité dans son champ aux hypothèses de conflits d'intérêt d'une part, et au signalement de délits et crimes d'autre part. Toutes deux intervenues dans l'« urgence émotionnelle » générée par l'affaire "Cahuzac », ces lois ont toutefois en commun de faire suite à une réflexion plus large des pouvoirs publics en la matière.

Le législateur semble ainsi avoir fait sienne la logique promue en matière de conflits d'intérêts par la Commission de réflexion pour la prévention des conflits d'intérêts dans la vie publique, présidée par le vice-président du Conseil d'État Jean-Marc Sauvé. Constatant l'absence de dispositifs d'alerte internes et propres à l'administration, la Commission proposait la mise en place d'un tel dispositif, afin que les « acteurs publics ») aient « la possibilité de déclencher cette alerte dès lors qu'ils constateraient qu'un conflit d'intérêts, potentiel ou avéré, est susceptible de soulever des difficultés pour l'institution qu'ils servent " (Pour une nouvelle déontologie de la vie publique, Rapport de la Commission de réflexion pour la prévention des conflits d'intérêts dans la vie publique, remis au Président de la République le 26 janvier 2011, p. 88). Le système que cette Commission appelait de ses vœux ne proposait en effet comme destinataires du signalement que les supérieurs hiérarchiques ou déontologues ad hoc des institutions de rattachement de l'acteur public en cause. Le rapport Pour un renouveau démocratique de la Commission sur la rénovation et la déontologie de la vie publique (rapport « Jospin ») se montrera quant à lui un peu plus ambitieux. En effet, la proposition $n^{\circ} 35$ de ce rapport, relative au dispositif d' "alerte éthique », avait pour but de renforcer le rôle des déontologues, revendiquant même le caractère "ouvert » du dispositif d'alerte. Toutefois, cette dernière demeurait somme toute assez silencieuse quant aux dispositifs de protection à mettre en œuvre au bénéfice des lanceurs d'alerte " placés dans une relation de dépendance quelconque à l'égard des personnes publiques concernées ».Ce n'était pourtant pas la position qui avait été adoptée par la commission "Sauvé », qui exigeait que le déclencheur d'alerte se trouve dans une situation de "conflit de devoirs ». De la même manière, la Commission nationale consultative des droits de l'homme, dans son Avis sur la probité de la vie publique du 27 juin 2013, demeurait tout aussi silencieuse quant aux aspects pratiques de la protection qu'elle appelait de ses vœux.

Mettant en partie en œuvre les préconisations des commissions précitées, les lois diffèrent toutefois en ce qui concerne l'étendue des personnes protégées et des canaux de signalement offerts aux lanceurs d'alerte. Ainsi, en matière de conflits d'intérêts, la loi du 11 octobre 2013 adopte une définition « ouverte » de la personne du lanceur d'alerte. En effet, ce dernier est défini comme étant la " personne » signalant une situation de conflits d'intérêts (aux termes de l'alinéa premier de l'article 2 de la loi du 11 octobre 2013, de " toute situation d'interférence entre un intérêt public et des intérêts publics ou privés qui est de nature à influencer ou à paraitre influencer l'exercice indépendant, impartial et objectif d'une fonction ». A contrario, dans la loi du 6 décembre 2013, seuls les salariés et fonctionnaires « dans l'exercice de leurs fonctions » font l'objet d'une protection en matière de signalement d'un crime ou d'un délit. De même, s'agissant des destinataires du signalement, les deux lois procèdent à une différenciation: Le signalement prévu par l'article 25 de la loi du 11 octobre 2013 en matière de conflits d'intérêts apparaît très encadré. Les destinataires sont en effet limitativement 
énumérés : l'employeur, le déontologue de l'organisme ou une « association de lutte contre la corruption agréée » selon les conditions fixées à l'article 1 du décret n 2014-327 du 12 mars 2014 relatif aux conditions d'agrément des associations de lutte contre la corruption en vue de l'exercice des droits reconnus à la partie civile. A contrario, l'article de la loi du 6 décembre 2013 ne précisant pas à quelle autorité doit être transmise une alerte, l'on en déduit logiquement que celle-ci peut cheminer par tous moyens, y compris par le biais de la presse. La circulaire NOR JUSD1402112C du 23 janvier 2014 relative à la présentation de la loi n ${ }^{\circ}$ 2013-1117 du 6 décembre 2013 relative à la lutte contre la fraude fiscale et la grande délinquance économique et financière en prend acte, en mentionnant notamment, parmi les éventuels destinataires de signalements, la presse..

Il est toutefois à noter que le législateur semble avoir entendu privilégier les révélations faites au SCPC. Le lanceur d'alerte professionnel peut ainsi notamment, à la faveur de la création par l'article 36 de la loi du 6 décembre 2013 de l'article 40-6 du code de procédure pénale, être mis en relation "à sa demande" avec le service central de prévention de la corruption "lorsque l'infraction signalée entre dans le champ de compétence de ce service ». Cette dernière disposition, selon les termes même de la circulaire d'application du texte (Circulaire NOR JUSD1402112C du 23 janvier 2014 financière), n'est toutefois pas exclusive de l'action du ministère public, qui peut "parallèlement apprécier l'opportunité d'informer le SCPC de l'existence de ce signalement et, dans le respect du secret de l'enquête, de lui transmettre tous éléments de contexte utiles concernant les faits révélés et le contexte de la révélation ». Rappelons en outre que cette conception très large des canaux de l'alerte a donné lieu à des débats assez vifs entre députés et sénateurs. Ces derniers ont souhaité, lors de la seconde lecture du projet de loi au Sénat, limiter la protection prévue par le nouvel article 1132-3-3 du Code du travail (et, pour les fonctionnaires, de l'article 6 ter A de la Loi n ${ }^{\circ} 83-634$ du 13 juillet 1983 portant droits et obligations des fonctionnaires) aux personnes signalant les faits aux autorités judiciaires ou administratives, à l'exclusion des révélations faites aux médias ou à des entreprises concurrentes.

31 S'agissant de la protection des lanceurs d'alerte stricto sensu, le nouvel article 1132-3-3 du Code du travail (et, pour les fonctionnaires, de l'article 6 ter A de la Loi $\mathrm{n}$ - 83-634 du 13 juillet 1983 portant droits et obligations des fonctionnaires) et l'article 25 de la loi du 11 octobre 2013 instaurent une protection calquée sur le modèle des dispositifs suscités contre les agissements discriminatoires ou les faits de harcèlement. Ces articles prévoient tous deux en effet qu'« aucune mesure concernant notamment le recrutement, la titularisation, la formation, la notation, la discipline, la promotion, l'affectation et la mutation » ne peut être prise à l'égard d'un fonctionnaire ou d'un agent non titulaire de droit public, qui aurait «relaté ou témoigné, de bonne foi, de faits constitutifs d'un délit ou d'un crime dont il aurait eu connaissance dans l'exercice de ses fonctions ». Comme pour les dispositifs de lutte contre les discriminations, la disposition prévoit également un partage de la charge de la preuve en cas de litige, puisque "dès lors que la personne présente des éléments de fait qui permettent de présumer qu'elle a relaté ou témoigné de bonne foi de faits constitutifs d'un délit ou d'un crime » il incombe désormais à l'administration de "prouver que sa décision est justifiée par des éléments objectifs étrangers à la déclaration ou au témoignage de l'intéressé ».

On pourra toutefois regretter que ces protections ne prévoient pas, d'une part, la nullité du licenciement discriminatoire pour ce qui est des salariés et, d'autre part n'envisagent pas, dans le secteur public, l'hypothèse du non-renouvellement d'un 
contrat d'emploi. Enfin, conformément aux vœux exprimés par le rapport Pour une nouvelle déontologie de la vie publique de la Commission Sauvé, l'article 25-II de la loi du 25 octobre 2013 prévoit, à titre dissuasif, de punir les signalements effectués «de mauvaise foi ou avec l'intention de nuire ou avec la connaissance au moins partielle de l'inexactitude des faits rendus publics ou diffusés » des peines prévues pour l'infraction de dénonciation calomnieuse (article 226-10 du Code pénal). Ainsi, complétant un dispositif autrefois parcellaire, incomplet et illisible, ces deux lois n'ouvrent au mieux qu'une brèche étroite dans une culture administrative française encore empreinte de la culture du secret ${ }^{9}$.

\section{$3 \%$ - L'inadaptation des lois de protection des secrets des sources à la problématique du whistleblowing}

33 La protection du secret des sources journalistiques présente un intérêt particulier pour la problématique des lanceurs d'alerte. En effet, les journalistes représentent un des canaux privilégiés utilisés par les lanceurs d'alerte " externes » afin de mettre à disposition du public des informations constituant un sujet d'intérêt public, et nécessaires au débat démocratique. La protection du secret des sources est donc essentielle, et le Comité des ministres du Conseil de l'Europe a estimé à ce titre que «la protection de la relation professionnelle entre les journalistes et leurs sources est plus importante que la valeur réelle de l'information en question pour le public. Toute révélation d'une source peut avoir un effet inhibant sur les futures sources » (Recommandation $n^{\circ} \mathrm{R}(2000) 7$ sur le droit des journalistes de ne pas révéler leurs sources, \$5). Les modalités de protection du secret des sources se heurtent cependant à plusieurs écueils: celui de la définition du « journaliste d'une part, celui de la définition des restrictions possibles à la protection de ce secret d'autre part. En France, il a fallu attendre la loi no 2010-1 du 4 janvier 2010, dite « loi Dati », pour que la protection du secret des sources fasse l'objet d'un même ensemble législatif, sous l'influence de la jurisprudence de la Cour Européenne des Droits de l'Homme (A). La future loi sur la protection des sources journalistiques viendra par la suite combler partiellement les lacunes de la loi Dati, sans opérer de révolution en la matière (B).

\section{A - L'apport très mesuré de la loi « Dati » du 1er janvier 2010.}

34 Cette loi, la première à prendre comme objet exclusif la protection du secret des sources des journalistes, s'inscrit dans un contexte assez particulier. La France a effectivement été fréquemment condamnée par la Cour EDH de 1999 à 2002 en raison d'atteintes au secret des sources caractérisant une violation de l'article 10 de la Convention Européenne des Droits de l'Homme (Conv. EDH). La loi Dati avait ainsi comme objectif majeur la mise en conformité avec la jurisprudence, constante depuis l'arrêt Handyside c. R.U du 7 décembre 1976 (Cour EDH, 7 décembre 1976, Handyside c. Royaume-Uni, Req. n 5493/72) et surtout l'arrêt Goodwin c. R.U de 1996 (Cour EDH, 11 juillet 2002, Goodwin c. RU, Req. $n^{\circ}$ 28957/95), de la Cour de Strasbourg relative à l'article 10. Cette jurisprudence consacre effectivement la liberté d'expression comme étant «l'un des fondements essentiels d'une société démocratique [dont] la presse est le chien de garde », ce que la cour a réaffirmé cela à l'occasion de ses arrêts Observer et Guardian c. R.U du 26 novembre 1991 (Cour EDH, 26 novembre 1991, Observer et Guardian c. Royaume-Uni, Req. 
$\mathrm{n}^{\circ}$ 13585/88 ; Cour EDH, G.C. 14 septembre 2010, Sanoma Uitgevers B.V. c. Pays-Bas, Req. $\mathrm{n}$ -38224/03 - ADL du 14 septembre 2010).

$\mathrm{Au}$ fil des affaires mettant en cause l'article 10, la Cour EDH a ainsi placé la liberté de la presse au sommet de la liberté d'expression au nom du droit du public à recevoir de l'information sur des questions d'intérêt général. C'est effectivement ce dernier droit qu'il s'agit de pouvoir impérativement assurer. Forte de cela, la Cour a pu dire que «la protection du secret des sources journalistiques est l'une des pierres angulaires de la liberté de la presse » et que "l'absence d'une telle protection pourrait dissuader les sources journalistiques d'aider la presse à informer le public sur des questions d'intérêt général. En conséquence, la presse pourrait être moins à même de jouer son rôle de "chien de garde " et son aptitude à fournir des informations précises et fiables pourrait s'en trouver amoindrie " (in Goodwin c. R.U, opinion dissidente commune des juges Ryssdal, Bernhardt, Thor Vilhjálmsson, Matscher, Walsh, Sir John Freeland et Baka \$2).

C'est notamment dans ce cadre que la France a pu être condamnée par cette même Cour. Dans l'affaire Fressoz et Roire c. France du 21 janvier 1999 (Cour EDH, 21 janvier 1999, Fressoz et Roire c. France, req. n²9183/95), la Cour a estimé nécessaire qu'une «quelconque restriction à l'exercice de la liberté d'expression [se trouve] établie de manière convaincante ", ce qu'elle rappellera à l'occasion de son arrêt Colombani c. France du 25 juin 2002 (Cour EDH, 25 juin 2002, Colombani c. France, req. n 51279/99). Il est enfin à noter que la Cour EDH a également pour jurisprudence constante, depuis l'arrêt Goodwin c. R.U, de dissocier l'intérêt public des informations traitées et diffusées par un journaliste du besoin social impérieux que cet intérêt pourrait constituer, et qui justifierait une atteinte au secret des sources. Elle a également tendance, depuis ce même arrêt, à condamner systématiquement les États ayant porté atteinte au secret des sources dans le cadre d'une procédure pénale.

Prenant acte de cette jurisprudence, la loi du 4 janvier 2010 a ainsi modifié la loi du 29 juillet 1881 en y introduisant un article 2, qui s'attache à établir tout d'abord le fait que «le secret des sources des journalistes est protégé dans l'exercice de leur mission d'information $\mathbf{d u}$ public ", et à préciser dans un deuxième temps la définition de journaliste, les possibilités d'atteinte au secret des sources, la qualification de l'atteinte, et les manières d'apprécier cette atteinte au cour d'une procédure pénale. Un journaliste est ainsi défini comme «[...] toute personne qui, exerçant sa profession dans une ou plusieurs entreprises de presse, de communication au public en ligne, de communication audiovisuelle ou une ou plusieurs agences de presse, y pratique, à titre régulier et rétribué, le recueil d'informations et leur diffusion au public ». Si cette définition se veut large, il n'en demeure pas moins que bon nombre d'acteurs comme Reporters Sans Frontières (RSF) au même titre qu'un certain nombre de journalistes, ont critiqué son aspect restrictif. Cette définition n'englobe effectivement pas les journalistes pigistes, qui peuvent contribuer au recueil et à la diffusion d'informations sans pour autant le faire de manière régulière et/ou rétribuée. De même, le cas des blogueurs n'entre pas dans cette catégorie. Cependant, l'alinéa le plus controversé de cette loi demeure celui visant à entourer les conditions \& possibilités d'atteinte au secret des sources, rédigé comme suit: "Il ne peut être porté atteinte au secret des sources que si un impératif prépondérant d'intérêt public le justifie et si les mesures envisagées sont strictement nécessaires et proportionnées au but légitime poursuivi ». Ces notions d'intérêt public prépondérant, de proportionnalité, de nécessité et de légitimité ont été critiquées en raison de la trop grande marge d'appréciation qu'elles laissent aux juridictions. 

affirmé les principes de nécessité et de proportionnalité des mesures engagées et d'identification d'un impératif prépondérant d'intérêt public dans un arrêt du 6 décembre 2011 (Cass. Crim., 6 décembre 2011, req. n 11-83970 - ADL du 10 décembre 2011 par 0 . Bachelet), dans le cadre de la réquisition de factures téléphoniques pour identifier les sources de journalistes travaillant sur la procédure judiciaire alors en cours de l' affaire Woerth-Bettencourt. Selon la Cour, ces conditions n'étaient, en l'espèce, pas réunies. Cependant, dans un arrêt du 14 mai 2013, (Cass. Crim., 14 mai 2013, req. $n^{\circ}$ 11-86626) cette même Cour a paradoxalement censuré l'arrêt d'une chambre de l'instruction ayant repris le même raisonnement que celui de l'arrêt précité pour annuler les réquisitions de factures détaillées («fadettes») prises aux fins d'identifier les destinataires des communications des journalistes. La Cour de cassation a estimé en l'espèce que la cour d'appel aurait dû préciser l'absence d'impératif prépondérant d'intérêt public considérant les circonstances de l'affaire, et caractériser davantage l'absence de nécessité et de proportionnalité des mesures. La notion d'intérêt public a également été placée au centre d'un arrêt du 4 juin 2013 de la Chambre de l'Instruction de Paris, interprétant cette notion très différemment de la Cour de cassation dans l'arrêt précité du 6 décembre 2011. En effet, selon la chambre de l'instruction en effet, l'intérêt public nécessitait en l'espèce que les journalistes soient poursuivis pour recel de violation du secret de l'instruction, l'atteinte au secret des sources ayant permis d'identifier l'informateur en question, à savoir un fonctionnaire de police que la chambre d'instruction a estimé être lié au secret et ne devoir en aucun cas y porter atteinte. La Cour de cassation a quant à elle cassé cet arrêt dans un jugement du 25 février 2014 ( Cass. Crim., 25 février 2014, req. $\mathrm{n}^{\circ}$ 13-84761).

40 On imagine par conséquent dans quel flou juridique les lanceurs d'alerte diffusant leurs informations par le biais de journalistes peuvent se trouver, considérant les divergences d'interprétation des critères précités. Si les débats parlementaires relatifs à la loi « Dati »font état d'une volonté du législateur de prévenir l'opposition du secret des sources dans le cadre d'affaires de terrorisme et de criminalité organisée par le biais de ces critères, cela ne s'est pas traduit précisément dans le texte de loi lui-même (rapport de Mme Marie-Anne Chapdelaine, au nom de la Commission des Lois de l'Assemblée Nationale, du 11 décembre 2012). D'autres dispositions permises par la loi «Dati» peuvent être critiquées, comme la modification de l'article 35 de la loi du 29 juillet 1881, instituant certes une immunité en matière de recel de violation du secret de l'instruction pour les journalistes notamment, mais uniquement lorsqu'ils se trouvent accusés de diffamation.

41 Si la loi du 4 janvier 2010 peut donc être saluée en ce qu'elle constitue une première mouture de la prise en compte juridique de la nécessaire protection du secret des sources des journalistes, elle demeure néanmoins incomplète et polysémique, en dépit des orientations interprétatives proposées par la Cour $\mathrm{EDH}^{10}$ au fur et à mesure de sa jurisprudence. Considérant cela, le gouvernement a souhaité mettre en œuvre une nouvelle loi, actuellement à l'état de projet ; mais il s'avère d'ores et déjà décevant. 


\section{B - Le projet de loi « renforçant la protection du secret des sources des journalistes » du 12 juin 2013, une actualisation en demi-teinte de la loi Dati}

\section{en raison de l'aspect disproportionné des mesures de perquisitions et saisies menées au domicile de plusieurs journalistes et au sein des rédactions des journaux} Le Point et L'Équipe. Considérant que la chambre de l'instruction n'avait pas identifié de besoin social impérieux, la Cour EDH a rappelé que «le droit des journalistes de taire leurs sources ne saurait être considéré comme un simple privilège qui leur serait accordé ou retiré en fonction de la licéité ou de l'illicéité des sources, mais un véritable attribut du droit à l'information, à traiter avec la plus grande circonspection » (\$124 de l'arrêt). La Cour de Strasbourg a ainsi rappelé sa jurisprudence constante, tout en insistant sur l'importance cruciale, pour la liberté de la presse ainsi que pour le débat démocratique, du droit des journalistes à protéger leurs sources dans la grande majorité des situations; sauf en cas d'infractions particulièrement graves ${ }^{11}$.

45 L'élaboration de ce projet de loi s'inscrit également dans le cadre d'affaires médiatiques notoires ayant défrayé la chronique, comme par exemple l'affaire des «fadettes » du Monde. Le projet de loi propose par conséquent un certain nombre de modifications de la loi « Dati » afin d'améliorer la protection des sources des journalistes : l'article 2 de la loi du 29 juillet 1881 a été modifié dans le sens d'une suppression de la nécessité d'exercer l'activité de journaliste de manière régulière et en tant que salarié, mais laisse intacte la notion de rétribution financière. Le projet de loi prévoit également la mise en œuvre du second principe de la recommandation du Conseil des Ministres du Conseil de l'Europe du 8 mars 2000 en étendant la protection du secret des sources aux collaborateurs de la rédaction. Cette prise d'acte se retrouve de manière prégnante dans la formulation de l'objet du projet de loi, la commission des lois ayant effectivement estimé que le but poursuivi par la protection du secret des sources n'était pas seulement de garantir «l'exercice de la mission d'information des journalistes » comme c'était le cas pour la loi du 4 janvier 2010, mais de garantir «l'information du public» (voir : rapport de Mme 
Marie-Anne Chapdelaine, au nom de la Commission des Lois de l'Assemblée Nationale, du 11 décembre 2012 cf. supra).

D'autres avancées majeures peuvent être soulignées, comme l'élaboration d'une définition plus précise des motifs permettant de porter atteinte au secret des sources, à savoir: la prévention ou la répression d'un crime, la prévention du délit d'atteinte à la personne humaine, et la prévention des délits d'atteinte aux intérêts fondamentaux de la Nation et de terrorisme. Ce dernier motif est toujours critiqué en raison de sa trop large interprétation, comme l'a rappelé RSF dans ses recommandations du 4 décembre 2013. Une avancée considérable a été faite concernant la supervision de ces atteintes, que le projet de loi confie au juge des libertés et de la détention (JLD), ce qui n'a pas été sans soulever plusieurs oppositions ou incompréhensions à l'occasion des débats parlementaires (voir la contribution de M. S. Hyughe, co-rapporteur du texte, p. 35-36-37 du rapport de Mme Marie-Anne Chapdelaine, au nom de la Commission des Lois de l'Assemblée Nationale, du 11 décembre 2012). En termes pénaux, le projet de loi vise à créer non pas un délit autonome en cas d'atteintes illégales au secret des sources, mais plusieurs circonstances aggravantes. Les peines d'amende pour la violation du domicile, du secret des correspondances ou d'intrusion dans un fichier informatique, en cas d'intention de porter atteinte au secret des sources, se verront ainsi aggravées. Sur proposition de la CNCDH, saisie d'un avis du 25 avril 2013 sur ce projet de loi par le gouvernement (CNCDH, Avis du 25 avril 2013 sur la réforme de la protection du secret des sources - ADL du 28 avril 2013 par A. Sireyjol), créer des circonstance aggravantes a pour avantage de «sanctuariser le principe de protection du secret des sources en évitant la multiplication d'infractions autonomes et en préservant la valeur symbolique de l'infraction » ( $\$ 20$ de l'avis). Enfin, et suite à l'arrêt Ressiot et autres c. France notamment, le projet de loi prévoit la création d'une immunité pénale pour le journaliste en cas de détention de documents en violation du secret de l'instruction, du secret professionnel ou de l'intimité de la vie privée.

Il est important de souligner ici l'influence qu'a pu avoir sur l'élaboration de ce projet la législation belge. En effet, la loi relative à la protection des sources des journalistes du 7 avril 2005, fait ainsi office de modèle ou de repoussoir, selon les conceptions, en termes de protection des sources. Cette loi est en effet représentative, d'une part, de la latitude qu'il est possible de donner à la protection du secret des sources, mais également des grandes divergences qui entourent ce sujet. Les débats parlementaires à la Chambre des représentants de Belgique ont effectivement opposé deux conceptions. L'une, proche de l'avis divergent du co-rapporteur du projet de loi français Sébastien Huyghe, vise à estimer trop fluctuant, imprécis et potentiellement mensonger le blogging \& journalisme "citoyen" effectué sur Internet. L'autre, considérant -avec Cédric Michalski, avocat au barreau de Mulhouse, chargé d'enseignement au centre universitaire d'enseignement du journalisme de Strasbourg- que la liberté de la presse est un droit politique qui devrait "être accordé à toute personne participant à la diffusion, collecte, production ou la rédaction d'informations" car elle "permet au citoyen de participer à la puissance publique et d'exercer indirectement sa souveraineté " (In rapport de Mme MarieAnne Chapdelaine, au nom de la Commission des Lois de l'Assemblée Nationale, du 11 décembre 2012, p13-14). Semblent effectivement être ignorées les règles déontologiques régissant la profession, selon lesquelles un journaliste se doit d'évaluer scrupuleusement la véracité et la vraisemblance des propos qu'il rapporte et traite ; sans quoi sa responsabilité pourrait donc être mise en cause. En dépit de cela, 
bon nombre de députés belges semblaient alors être extrêmement réticents à l'extension du secret des sources aux personnes désignées à l'article 2, car cela permettrait l'ouverture aux médias en ligne et à leur contenu.

48 C'est notamment ce que soulève RSF dans ses recommandations, rappelant la définition du journaliste établie par l'Observation Générale $\mathrm{N}^{\circ} 34$ du Comité des droits de l'Homme des Nations Unies de juillet 2011 selon laquelle le journalisme est « une fonction exercée par des personnes de tous horizons, notamment les reporters et analystes professionnels à plein temps, ainsi que les blogueurs et autres particuliers qui publient eux mêmes le produit de leur travail, sous forme imprimée, sur l'Internet ou autrement » (in Observation Générale $\mathrm{N}^{\circ} 34$, § 44). L'on remarque ainsi l'insuffisance de ce projet de loi en termes de protection du secret des sources, en raison de termes peu précis et de l'absence de prise en compte des documentaristes et réalisateurs dans la définition de journaliste. La tâche n'est cependant pas aisée ; « la définition du concept de journaliste [devant] (...) traduire la recherche de l'équilibre entre, d'une part, la liberté d'expression, la liberté de la presse et le droit à l'information, et d'autre part le droit au respect de la vie privée " (Valérie Deom, in Rapport Wathelet pour la Commission de la Justice de l'Assemblée Nationale belge sur la protection du secret des sources, p. 29), ainsi que du secret professionnel et du secret de l'instruction.

Ainsi, si l'année 2013 a bien marqué une évolution encourageante des protections accordées aux lanceurs d'alerte, cette évolution n'en reste pas moins mesurée. Le droit positif actuel, constitué d'un amoncellement de législations adoptées sans cohérence d'ensemble, apparait bien peu compréhensible. A cet égard, la très récente publication par le Conseil de l'Europe d'une recommandation sur la protection des lanceurs d'alerte (Comité des Ministres, 30 avril 2014, Recommandation CM/Rec(2014)7 aux Etats membres sur la protection des lanceurs d'alerte) semble constituer, implicitement mais nécessairement, un appel du pied à la France et aux autres états européens n'accordant qu'une protection "partielle» aux porteurs d'alerte. Dans la mesure où les lanceurs d'alerte "peuvent contribuer à renforcer la transparence et la responsabilité démocratique », et donc l'effectivité des droits de l'homme et l'émergence d'une démocratie véritable, il s'agit de penser leur protection dans un cadre plus large que des lois sectorielles et dictées par l'urgence émotionnelle, pour que puisse émerger un véritable débat public sur cette problématique. Comme le souligne en effet Paul Stephenson, «plus le débat initial sur les protections accordées aux lanceurs d'alerte prendra appui sur le public, plus ces protections pourront être connues et respectées » (Paul Stephenson, « What makes a good whistleblowing law?»). Sans nous aventurer dans les méandres d'un discours prescriptif, pouvons-nous souligner qu'il serait sans doute temps de mettre en place les conditions d'émergence d'un tel débat en France. 
Les Lettres « Actualités Droits-Libertés » (ADL) du CREDOF (pour s'y abonner) sont accessibles sur le site de la Revue des Droits de l'Homme (RevDH) - Contact

\section{NOTES}

1. Sur cette notion l'on pourra consulter l'ouvrage suivant: V. Champeil-Desplats et D. Lochak (dir), "A la recherche de l'effectivité des droits de l'homme " Centre de recherches et d'études sur les droits fondamentaux Presses universitaires de Nanterre, 2007

2. Voir, notamment : Brown, A. J., "Flying Foxes and Freedom of Speech: Statutory Recognition of Public Whistleblowing in Australia.",Whistleblowing and Democratic Values (2011): 86 ; Elletta Sangrey Callahan, Terry Morehead Dworkin, and David Lewis, " Whistleblowing: Australian, UK, and US Approaches to Disclosure in the Public Interest.", Va. J. Int'l L. 44 (2003): 879

3. Sur le droit à la santé: Tatiana Gründler, «Le droit à la protection de la santé » in D. Roman, Droits des pauvres, pauvres droits. Recherche sur la justiciabilité des droits de l'homme, Rev. DH 1 (2012) : 371-387.

4. V. son témoignage dans un séminaire sur les lanceurs d'alerte le 27 janvier 2014 sur le blog les périphériques vous parlent... et la chaine youtube

5. Voir, sur ce sujet, le remarquable article de Danièle Lochak: Danièle Lochak, «La dénonciation, stade suprême ou perversion de la démocratie? ", in L'état de droit : mélanges en l'honneur de Guy Braibant, 1996, p. 451-470

6. Corinne Lepage, Mission Lepage, Rapport final phase 1. 2008) en faisant référence au Freedom of Information Act 2000 pour la Grande-Bretagne et le Freedom of Information Act (U.S.C. § 552. U.S. Government Law (2002)) pour les Etats-Unis

7. Voir, par ex: Cass. Soc., 4 février 1997, $\mathrm{n}^{\circ}$ 96-40678 : validation du licenciement de deux salariées qui avaient dénoncé des pratiques dangereuses pour la santé publique, dénonciation considérée comme participation "à une campagne de dénigrement contre leur employeur "

8. Pour un aperçu complet sur l'ensemble de cette thématique des " clauses de confidentialité »: Olivier Leclerc. "Sur la validité des clauses de confidentialité en droit du travail." Droit social 2 (2005): 173-180.

9. Jacques Chevallier, Le mythe de la transparence administrative, Information et transparence administratives, PUF, 1988

10. La Cour de Strasbourg a dégagé plusieurs critères permettant de déterminer la licéité d'une atteinte au secret des sources et venant éclairer la loi Dati :

- la nécessité de l'atteinte, qu'elle lie à l'importance de l'information recherchée pour prévenir ou réprimer une action

- la proportionnalité de l'atteinte, relativement à laquelle la Cour vérifiera notamment si d'autres mesures auraient pu permettre de parvenir aux mêmes résultats - Source : Comité des Ministres 
du Conseil de l'Europe, Recommandation $n^{\circ} \mathrm{R}(2000) 7$ sur le droit des journalistes de ne pas révéler leurs sources

11. La Cour EDH admet la limitation du secret des sources afin de prévenir des infractions particulièrement graves, - si l'ingérence s'avère néanmoins proportionnée comme ce fut le cas lors d'une affaire dont elle eu à connaître concernant un reportage d'un journaliste danois sur des réseaux pédophiles. Considérant qu'il s'agissait en l'espèce d'un impératif prépondérant d'intérêt général, la Cour a conclut à l'absence de violation de l'article 10 dans son arrêt Nordisk Film \& TV A/S c. Danemark du 8 décembre 2005.

\section{RÉSUMÉS}

L'année 2013 aura été marquée par une étape historique dans la mise en place d'un statut spécifique pour les lanceurs d'alerte. En effet, trois lois successives ont mis en place, dans des domaines différents, une protection pour les personnes soucieuses d'exprimer des «avertissements de bonne foi concernant divers actes illicites, y compris toutes les violations graves des droits de l'homme, qui affectent ou menacent la vie, la santé, la liberté et tout autre intérêt légitime des individus. Cette protection reste toutefois très parcellaire et restrictive. A cet égard, la très récente publication par le Comité des Ministres du Conseil de l'Europe d'une recommandation sur la protection des lanceurs d'alerte, en date du 30 avril 2014, vient rappeler avec acuité à quel point la France reste en retrait au regard des standards internationaux et européens en matière de protection des donneurs d'alerte.

\section{AUTEURS}

\section{ANNA BILLARD}

Etudiante du Master 2 "Droits de l'Homme" de l'Université de Paris Ouest Nanterre La Défense

\section{MARC DURANTON}

Etudiant du Master 2 "Droits de l'Homme" de l'Université de Paris Ouest Nanterre La Défense JEAN-PHILIPPE FOEGLE

Etudiant du Master 2 "Droits de l'Homme" de l'Université de Paris Ouest Nanterre La Défense TRISTAN MARTIN-TEODORCZYK

Etudiant du Master 2 "Droits de l'Homme" de l'Université de Paris Ouest Nanterre La Défense 